\title{
On the average order of the lattice rest of a convex body
}

\author{
by \\ WolfGang MüLler (Graz)
}

1. Introduction. Let $\mathcal{B}_{s}$ denote a compact convex subset of $\mathbb{R}^{s}, s \geq 2$, which contains the origin as an inner point. Suppose that the boundary $\partial \mathcal{B}_{s}$ of $\mathcal{B}_{s}$ is an $(s-1)$-dimensional surface of class $C^{6 s+13}$ with finite nonzero Gaussian curvature throughout. For $x>0$ define $A_{\mathcal{B}_{s}}(x)$ as the number of points of the lattice $\mathbb{Z}^{s}$ in the "blown up" domain $x \mathcal{B}_{s}$, i.e. $A_{\mathcal{B}_{s}}(x)=$ $\#\left(x \mathcal{B}_{s} \cap \mathbb{Z}^{s}\right)$, and $P_{\mathcal{B}_{s}}(x)$ as the "lattice rest" $\left.{ }^{1}\right)$

$$
P_{\mathcal{B}_{s}}(x)=A_{\mathcal{B}_{s}}(x)-\operatorname{vol}\left(\mathcal{B}_{s}\right) x^{s} .
$$

For such a general convex body $\mathcal{B}_{s}$ E. Hlawka [3] proved that

$$
P_{\mathcal{B}_{s}}(x)=O\left(x^{s(s-1) /(s+1)}\right)
$$

and

$$
P_{\mathcal{B}_{s}}(x)=\Omega\left(x^{(s-1) / 2}\right) .
$$

In the last years both estimates have been improved (see [11, 12, 16-18] and for planar domains $[5,15])$.

In the present article we study the mean square of $P_{\mathcal{B}_{s}}(x)$. For planar domains this problem has been attacked by W. G. Nowak [14] who proved

$$
\int_{0}^{X}\left|P_{\mathcal{B}_{2}}(x)\right|^{2} d x=O\left(X^{2}\right)
$$

This bound is best possible since in the special case of a cirlce $\mathcal{S}_{2}$, say, it

1991 Mathematics Subject Classification: Primary 11P21.

Key words and phrases: lattice points.

$\left({ }^{1}\right)$ Note that many authors use $t=x^{2}$ as parameter. This means that the domain $\mathcal{B}_{s}$ is "blown up" by the factor $x=\sqrt{t}$. 
can be replaced by the asymptotic formula (see I. Kátai [10] $\left({ }^{2}\right)$ )

$$
\int_{0}^{X}\left|P_{\mathcal{S}_{2}}(x)\right|^{2} d x=c_{2} X^{2}+O\left(X(\log X)^{2}\right) \quad\left(c_{2}>0\right) .
$$

For $s \geq 3$ Nowak's approach does not give satisfactory bounds. To see what one should expect in higher dimensions let us consider the case of the $s$-dimensional unit ball $\mathcal{S}_{s}$. This case (together with the case of rational ellipsoids) is particularly well understood. For $s \geq 4$, A. Walfisz [20] proved

$$
\int_{0}^{X}\left|P_{\mathcal{S}_{s}}(x)\right|^{2} d x=c_{s} X^{2 s-3}+O\left(R_{s}\right) \quad\left(c_{s}>0\right),
$$

where $R_{s}=X^{2 s-5}$ if $s>5, R_{5}=X^{5}(\log X)^{2}$ and $R_{4}=X^{4} \log X$. In the case $s=3$, V. Jarník [9] obtained the asymptotic formula

$$
\int_{0}^{X}\left|P_{\mathcal{S}_{3}}(x)\right|^{2} d x=c_{3} X^{3} \log X+O\left(X^{3}(\log X)^{1 / 2}\right) \quad\left(c_{3}>0\right) .
$$

The proof of both results uses the fact that the generating function of the number of lattice points on the sphere is a theta function. It therefore cannot be carried over to our general situation. Using a different method we prove the following higher dimensional analogue to (2).

TheOREM. Let $\mathcal{B}_{s}$ denote a compact convex subset of $\mathbb{R}^{s}, s \geq 4$, which contains the origin as an inner point. Suppose that the boundary $\partial \mathcal{B}_{s}$ of $\mathcal{B}_{s}$ is an $(s-1)$-dimensional surface of class $C^{6 s+13}$ with finite nonzero curvature throughout. Then for every fixed $\varepsilon>0$,

$$
\int_{0}^{X}\left|P_{\mathcal{B}_{s}}(x)\right|^{2} d x \ll X^{2 s-3+\varepsilon} .
$$

The theorem shows that spherical balls belong to those smooth convex bodies with nonzero curvature which have (on average) large lattice rest. In general the lattice rest can be much smaller. For instance, V. Jarník [8] proved that for $s \geq 4$ almost all ellipsoids

$$
\mathcal{E}_{s}(a)=\left\{\left(x_{1}, \ldots, x_{s}\right) \in \mathbb{R}^{s}: \sum_{i=1}^{s} a_{i} x_{i}^{2} \leq 1\right\} \quad\left(a=\left(a_{1}, \ldots, a_{s}\right) \in \mathbb{R}_{+}^{s}\right)
$$

(in the sense of Lebesgue) satisfy $P_{\mathcal{E}_{s}(a)}(x)=O\left(x^{s / 2+\varepsilon}\right)$ for every $\varepsilon>0$.

The method used to prove (5) seems to be new. Starting from a trivial bound for the mean square of $P_{\mathcal{B}_{s}^{*}}(x)$, where $\mathcal{B}_{s}^{*}$ denotes the polar body of

$\left({ }^{2}\right)$ Actually Kátai considers the mean square of $P_{\mathcal{S}_{2}}(\sqrt{t})$. Using integration by parts it is easy to see that his formulation is equivalent to ours. The same remark applies to (3) and (4). 
$\mathcal{B}_{s}$, it produces a nontrivial bound for the mean square of $P_{\mathcal{B}_{s}}(x)$. Since $\mathcal{B}_{s}^{* *}=\mathcal{B}_{s}$ the same method applied to $\mathcal{B}_{s}^{*}$ gives a better bound for the mean square of $P_{\mathcal{B}_{s}^{*}}(x)$. Iterating this process yields (5). Unfortunately, the iteration process produces better bounds only if $s \geq 4$. The interesting case $s=3$ remains open.

2. Geometrical preliminaries. Let $\mathcal{B}_{s}$ denote a compact convex subset of $\mathbb{R}^{s}, s \geq 2$, which contains the origin as an inner point. The polar body $\mathcal{B}_{s}^{*}$ of $\mathcal{B}_{s}$ is defined by

$$
\mathcal{B}_{s}^{*}=\left\{x \in \mathbb{R}^{s}: \sup _{y \in \mathcal{B}_{s}}\langle x, y\rangle \leq 1\right\},
$$

where $\langle\cdot, \cdot\rangle$ denotes the standard scalar product in $\mathbb{R}^{s}$. The polar body of a compact convex body which contains the origin as an inner point is again a compact convex body which contains the origin as an inner point. Furthermore, $\mathcal{B}_{s}^{* *}=\mathcal{B}_{s}$ (cf. [13], p. $65 \mathrm{ff}$ ). In order to apply the iterative process simultaneously to $\mathcal{B}_{s}$ and $\mathcal{B}_{s}^{*}$ we have to check that, if $\partial \mathcal{B}_{s}$ is smooth with nonzero curvature throughout, then the same is true for $\partial \mathcal{B}_{s}^{*}$. The following lemma is a multi-dimensional analogue of Huxley [6], Lemma 4, where only planar domains are considered.

Lemma 1. Let $x=x(t), t=\left(t_{1}, \ldots, t_{s-1}\right)$, be a regular (local) parametrization of $\partial \mathcal{B}_{s}$ of class $C^{k}, k \geq 2$, and $u=u(t)$ the unit outward normal vector of the tangent hyperplane at $x(t)$. Suppose that the Gaussian curvature $\kappa(x)$ of $\partial \mathcal{B}_{s}$ at $x$ is nonzero. Then

$$
x^{*}(t)=\langle x(t), u(t)\rangle^{-1} u(t)
$$

is a regular (local) parametrization of $\partial \mathcal{B}_{s}^{*}$ of class $C^{k-1}$ and

$$
\left|\kappa^{*}\left(x^{*}\right) \kappa(x)\right|=\left(\|x\| \cdot\left\|x^{*}\right\|\right)^{-s-1},
$$

where $\kappa^{*}\left(x^{*}\right)$ denotes the Gaussian curvature of $\partial \mathcal{B}_{s}^{*}$ at $x^{*}$. Furthermore, the spherical map of $\partial \mathcal{B}_{s}$ (e.g. the map which sends a point $x$ of $\partial \mathcal{B}_{s}$ to the endpoint of the outward normal vector $u$ on the unit sphere $\left.\partial \mathcal{S}_{s}\right)$ and the spherical map of $\partial \mathcal{B}_{s}^{*}$ are one-to-one and of class $C^{k-1}$.

Pr o of. Here regular means that the vectors $v_{i}=\partial x / \partial t_{i}, 1 \leq i \leq s-1$, form a basis of the $(s-1)$-dimensional tangent space $\mathcal{T}_{x}$ at $x$. Then $u$ is (as a function of $v_{i}, 1 \leq i \leq s-1$ ) of class $C^{k-1}$. From the fact that the pole of every tangent hyperplane (with respect to the unit sphere) is a point on $\partial \mathcal{B}_{s}^{*}$ (cf. [13], p. 67) we obtain

$$
x^{*}=x^{*}(t)=\langle x, u\rangle^{-1} u \in \partial \mathcal{B}_{s}^{*} .
$$

This shows that $x^{*}$ is of class $C^{k-1}$. Note that $\langle x, u\rangle>0$ since the origin is an inner point of $\mathcal{B}_{s}$. 
The Gaussian curvature $\kappa(x)$ of $\partial \mathcal{B}_{s}$ at $x$ is defined as the determinant of the linear map $L_{x}: \mathcal{T}_{x} \rightarrow \mathcal{T}_{x}$ which maps $v \in \mathcal{T}_{x}$ to $L_{x}(v)=\nabla_{v} u$, where $\nabla_{v} u$ denotes the derivative of the normal vector $u$ with respect to the direction $v$. Its sign depends on the orientation of the surface $\partial \mathcal{B}_{s}$. The linear map is determined by $L_{x}\left(v_{i}\right)=\partial u / \partial t_{i} \in \mathcal{T}_{x}$. It can be extended linearly from $\mathcal{T}_{x}$ to the entire $s$-dimensional space by setting $L_{x}(u)=u$. This does not change the absolute value of the determinant. Hence, if the matrix $B \in \mathbb{R}^{s \times s}$ is defined by

$$
\left(\frac{\partial u}{\partial t_{1}}, \ldots, \frac{\partial u}{\partial t_{s-1}}, u\right)=B\left(v_{1}, \ldots, v_{s-1}, u\right),
$$

then $|\operatorname{det} B|=|\kappa(x)|>0$. It follows that $\partial u / \partial t_{1}, \ldots, \partial u / \partial t_{s-1}, u$ are linearly independent. This implies that the tangent vectors

$$
v_{i}^{*}=\frac{\partial x^{*}}{\partial t_{i}}=\langle x, u\rangle^{-1} \frac{\partial u}{\partial t_{i}}-\langle x, u\rangle^{-2}\left\langle x, \partial u / \partial t_{i}\right\rangle u \quad(1 \leq i \leq s-1)
$$

are linearly independent, proving that the parametrization $x^{*}(t)$ of $\partial \mathcal{B}_{s}^{*}$ is regular. Since $\left\langle v_{i}^{*}, x\right\rangle=0$ for $1 \leq i \leq s-1$, we find that $u^{*}=\|x\|^{-1} x$ is normal to the tangent space $\mathcal{T}_{x}^{*}$ of $\partial \mathcal{B}_{s}^{*}$ at $x^{*}$. Representing $u^{*}$ as

$$
u^{*}=\|x\|^{-1} x=\sum_{i=1}^{s-1} \frac{\alpha_{i}}{\langle x, u\rangle} \cdot \frac{\partial u}{\partial t_{i}}+\frac{\langle x, u\rangle}{\|x\|} u
$$

with some $\alpha_{i} \in \mathbb{R}$ we conclude that

$$
\begin{aligned}
u^{*} & =\sum_{i=1}^{s-1} \alpha_{i} v_{i}^{*}+\langle x, u\rangle^{-1}\left(\sum_{i=1}^{s-1} \alpha_{i} \frac{\left\langle x, \partial u / \partial t_{i}\right\rangle}{\langle x, u\rangle}+\frac{\langle x, u\rangle^{2}}{\|x\|}\right) u \\
& =\sum_{i=1}^{s-1} \alpha_{i} v_{i}^{*}+\frac{\|x\|}{\langle x, u\rangle} u
\end{aligned}
$$

Together with (7) and (8) this yields

$$
\begin{aligned}
\operatorname{det}\left(v_{1}^{*}, \ldots, v_{s-1}^{*}, u^{*}\right) & =\operatorname{det}\left(v_{1}^{*}, \ldots, v_{s-1}^{*}, \frac{\|x\|}{\langle x, u\rangle} u\right) \\
& =\frac{\|x\|}{\langle x, u\rangle} \operatorname{det}\left(\langle x, u\rangle^{-1} \frac{\partial u}{\partial t_{1}}, \ldots,\langle x, u\rangle^{-1} \frac{\partial u}{\partial t_{s-1}}, u\right) \\
& =\frac{\|x\|}{\langle x, u\rangle^{s}}(\operatorname{det} B) \operatorname{det}\left(v_{1}, \ldots, v_{s-1}, u\right) .
\end{aligned}
$$

If $B^{*} \in \mathbb{R}^{s \times s}$ denotes the matrix defined by

$$
\left(\frac{\partial u^{*}}{\partial t_{1}}, \ldots, \frac{\partial u^{*}}{\partial t_{s-1}}, u^{*}\right)=B^{*}\left(v_{1}^{*}, \ldots, v_{s-1}^{*}, u^{*}\right),
$$


then $\left|\operatorname{det} B^{*}\right|=\left|\kappa^{*}\left(x^{*}\right)\right|$. Moreover, since

$$
\frac{\partial u^{*}}{\partial t_{i}}=\frac{\partial}{\partial t_{i}}\left(\|x\|^{-1} x\right)=\|x\|^{-1} v_{i}-\|x\|^{-2}\left\langle x, v_{i}\right\rangle u^{*}
$$

we obtain

$$
\begin{aligned}
\operatorname{det}\left(\frac{\partial u^{*}}{\partial t_{1}}, \ldots, \frac{\partial u^{*}}{\partial t_{s-1}}, u^{*}\right) & =\|x\|^{-s} \operatorname{det}\left(v_{1}, \ldots, v_{s-1}, x\right) \\
& =\|x\|^{-s} \operatorname{det}\left(v_{1}, \ldots, v_{s-1},\langle x, u\rangle u\right)
\end{aligned}
$$

and

$$
\begin{aligned}
\left|\kappa^{*}\left(x^{*}\right)\right| & =\left|\operatorname{det}\left(\frac{\partial u^{*}}{\partial t_{1}}, \ldots, \frac{\partial u^{*}}{\partial t_{s-1}}, u^{*}\right)\right| \cdot\left|\operatorname{det}\left(v_{1}^{*}, \ldots, v_{s-1}^{*}, u^{*}\right)\right|^{-1} \\
& =\left(\frac{\langle x, u\rangle}{\|x\|}\right)^{s+1}|\kappa(x)|^{-1} .
\end{aligned}
$$

This proves (6). Finally, we note that the spherical map of the boundary of a compact convex domain with nonvanishing Gaussian curvature is always one-to-one (cf. [19], p. 105). It follows from the above discussion that the maps $x \mapsto u$ and $x^{*} \mapsto u^{*}$ are of class $C^{k-1}$.

Lemma 2. Let $\mathcal{B}_{s}$ be a compact convex subset of $\mathbb{R}^{s}, s \geq 2$, which contains the origin as an inner point. Assume that the spherical map of $\partial \mathcal{B}_{s}$ is one-to-one and of class $C^{2 s+12}$, and that the Gaussian curvature of $\partial \mathcal{B}_{s}$ is nonzero throughout. Then the Fourier transform of the indicator function $I_{x \mathcal{B}_{s}}$,

$$
\widehat{I}_{x \mathcal{B}_{s}}(k)=\int_{x \mathcal{B}_{s}} e^{2 \pi i\langle k, u\rangle} d u \quad\left(k \in \mathbb{R}^{s}\right),
$$

satisfies, for $k \neq 0$,

$$
\begin{aligned}
\widehat{I}_{x \mathcal{B}_{s}}(k)= & (2 \pi)^{-1} x^{(s-1) / 2}\|k\|^{-(s+1) / 2}\left(\alpha_{k} e^{2 \pi i x H(k)-\gamma i}+\alpha_{-k} e^{-2 \pi i x H(-k)+\gamma i}\right) \\
& +O\left(x^{(s-3) / 2}\|k\|^{-(s+3) / 2}\right),
\end{aligned}
$$

where $\gamma=(s+1) \pi / 4, \alpha_{k}=\sqrt{\kappa_{k}}\left(\kappa_{k}\right.$ denotes the Gaussian curvature of $\partial \mathcal{B}_{s}$ at the point where the outward normal has direction $k$ ) and $H$ denotes the distance function of the polar body $\mathcal{B}_{s}^{*}$.

Proof. This is a special case of Hlawka's Satz 2 in [4] (see also Satz 5 in [3]). Note that the distance function of $\mathcal{B}_{s}^{*}$ agrees with the "tag"-function of $\mathcal{B}_{s}$ (cf. [13], p. 127) and that $\alpha_{k} \asymp 1$.

3. The basic estimate of $P_{\mathcal{B}_{s}}(x)$. As usual it is easier to estimate a smoothed version of $P_{\mathcal{B}_{s}}$ rather than $P_{\mathcal{B}_{s}}$ itself. To this purpose we introduce a weight function $\delta_{1}: \mathbb{R}^{s} \rightarrow[0, \infty)$ which has the following properties (see [1], p. 88 , or [7] for the existence of such a function): 
(i) the support of $\delta_{1}$ lies inside the unit ball $\mathcal{S}_{s}$,

(ii) $\int_{\mathbb{R}^{s}} \delta_{1}(y) d y=1$,

(iii) the Fourier transform $\widehat{\delta}_{1}(k)=\int_{\mathbb{R}^{s}} \delta_{1}(y) e^{2 \pi i\langle k, y\rangle} d y, k \in \mathbb{R}^{s}$, satisfies $\widehat{\delta}_{1}(k) \ll \exp \left(-\|k\|^{1 / 2}\right)$.

For $0<\varepsilon<1$ set $\delta_{\varepsilon}(y)=\varepsilon^{-s} \delta_{1}\left(\varepsilon^{-1} y\right), y \in \mathbb{R}^{s}$, then

$$
\operatorname{supp}\left(\delta_{\varepsilon}\right) \subseteq \varepsilon \mathcal{S}_{s}, \quad \widehat{\delta}_{\varepsilon}(0)=1, \quad \widehat{\delta}_{\varepsilon}(k)=\widehat{\delta}_{1}(\varepsilon k) \ll \exp \left(-(\varepsilon\|k\|)^{1 / 2}\right) .
$$

We use convolution with $\delta_{\varepsilon}$ to smooth the indicator function $I_{x \mathcal{B}_{s}}$ :

$$
I_{x \mathcal{B}_{s}} * \delta_{\varepsilon}(z)=\int_{\mathbb{R}^{s}} I_{x \mathcal{B}_{s}}(y) \delta_{\varepsilon}(z-y) d y \quad\left(z \in \mathbb{R}^{s}\right) .
$$

LEMMA 3. Let $\mathcal{B}_{s}$ be a compact convex subset of $\mathbb{R}^{s}$ which contains the origin as an inner point. Denote by $\varrho>0$ the radius of any inscribed ball $\varrho \mathcal{S}_{s} \subseteq \mathcal{B}_{s}$ and set $x_{ \pm}=x \pm \varepsilon / \varrho>0$. Then for all $z \in \mathbb{R}^{s}$,

$$
I_{x_{-} \mathcal{B}_{s}} * \delta_{\varepsilon}(z) \leq I_{x \mathcal{B}_{s}}(z) \leq I_{x_{+} \mathcal{B}_{s}} * \delta_{\varepsilon}(z) .
$$

Proof. We first claim that for $d \geq 0$,

$$
y \in \mathcal{B}_{s}, \quad z \notin(1+d) \mathcal{B}_{s} \Rightarrow\|z-y\|>\varrho d .
$$

To prove this (geometrically evident) fact consider the distance function $F(u)=\inf \left\{\lambda: u \in \lambda \mathcal{B}_{s}\right\}$ of $\mathcal{B}_{s}$. Then $y \in \mathcal{B}_{s}$ if and only if $F(y) \leq 1$, and $z \notin(1+d) \mathcal{B}_{s}$ if and only if $F(z)>1+d$. Since the distance function of a convex body is convex we conclude $F(z)=F(z-y+y) \leq F(z-y)+F(y)$. This implies $F(z-y)>d$. Hence $z-y \notin d \mathcal{B}_{s} \supseteq \varrho d \mathcal{S}_{s}$, and this proves (10). Since $0 \leq I_{x_{ \pm} \mathcal{B}_{s}} * \delta_{\varepsilon}(x) \leq 1$ the lemma follows if we can show that

$$
\begin{aligned}
\text { (i) } z \notin x \mathcal{B}_{s} \Rightarrow I_{x_{-} \mathcal{B}_{s}} * \delta_{\varepsilon}(z)=0, \\
\text { (ii) } z \in x \mathcal{B}_{s} \Rightarrow I_{x_{+} \mathcal{B}_{s}} * \delta_{\varepsilon}(z)=1 .
\end{aligned}
$$

To prove (i) assume that $z \notin x \mathcal{B}_{s}$ and $y \in x_{-} \mathcal{B}_{s}$; then $z=x z_{0}$ with $z_{0} \notin \mathcal{B}_{s}$, $y=x_{-} y_{0}$ with $y_{0} \in \mathcal{B}_{s}$ and by $(10)$

$$
\|z-y\|=x_{-}\left\|\left(1+\frac{\varepsilon}{\varrho x_{-}}\right) z_{0}-y_{0}\right\|>\varepsilon .
$$

Hence, for every $y \in \mathbb{R}^{s}, I_{x_{-} \mathcal{B}_{s}}(y) \delta_{\varepsilon}(z-y)=0$. This implies $I_{x_{-} \mathcal{B}_{s}} * \delta_{\varepsilon}(z)=$ 0 . The proof of (ii) is similar. Note that the right hand side in (ii) is equivalent to $I_{\left(x_{+} \mathcal{B}_{s}\right)^{C}} * \delta_{\varepsilon}(z)=0$.

We are now in a position to derive the basic estimate of $P_{\mathcal{B}_{s}}(x)$. By Lemma 3,

$$
\sum_{k \in \mathbb{Z}^{s}} I_{x_{-} \mathcal{B}_{s}} * \delta_{\varepsilon}(k) \leq A_{\mathcal{B}_{s}}(x) \leq \sum_{k \in \mathbb{Z}^{s}} I_{x_{+} \mathcal{B}_{s}} * \delta_{\varepsilon}(k)
$$


The multi-dimensional Poisson summation formula (see [2]) yields

$$
\sum_{k \in \mathbb{Z}^{s}} \widehat{I}_{x_{-} \mathcal{B}_{s}}(k) \widehat{\delta}_{\varepsilon}(k) \leq A_{\mathcal{B}_{s}}(x) \leq \sum_{k \in \mathbb{Z}^{s}} \widehat{I}_{x_{+} \mathcal{B}_{s}}(k) \widehat{\delta}_{\varepsilon}(k) .
$$

Since $\widehat{\delta}_{\varepsilon}(0)=1$ and

$$
\widehat{I}_{x_{ \pm} \mathcal{B}_{s}}(0)=\operatorname{vol}\left(\mathcal{B}_{s}\right) x_{ \pm}^{s}=\operatorname{vol}\left(\mathcal{B}_{s}\right) x^{s}+O\left(x^{s-1} \varepsilon\right)
$$

we obtain

$$
P_{\mathcal{B}_{s}}(x) \ll\left|\sum_{k \in Z_{*}^{s}} \widehat{I}_{x_{-} \mathcal{B}_{s}}(k) \widehat{\delta}_{\varepsilon}(k)\right|+\left|\sum_{k \in Z_{*}^{s}} \widehat{I}_{x_{+} \mathcal{B}_{s}}(k) \widehat{\delta}_{\varepsilon}(k)\right|+x^{s-1} \varepsilon,
$$

where $Z_{*}^{s}=\mathbb{Z}^{s} \backslash\{0\}$ for short. In the right hand side we insert the asymptotic expansion of Lemma 2. For $s \geq 4, X \leq x \leq 2 X$ and $\varepsilon \geq X^{-1}$ the contribution of the error term is at most

$$
X^{(s-3) / 2} \sum_{k \in Z_{*}^{s}}\|k\|^{-(s+3) / 2}\left|\widehat{\delta}_{\varepsilon}(k)\right| \ll\left(X \varepsilon^{-1}\right)^{(s-3) / 2} \ll X^{s-1} \varepsilon .
$$

Here we used the estimate

$$
\begin{aligned}
\sum_{k \in Z_{*}^{s}}\|k\|^{-\alpha} \exp \left(-(\varepsilon\|k\|)^{1 / 2}\right) & =\int_{1 / 2}^{\infty} u^{-\alpha} \exp \left(-(\varepsilon u)^{1 / 2}\right) d A_{\mathcal{S}_{s}}(u) \\
& \ll \varepsilon^{\alpha-s},
\end{aligned}
$$

which is valid for $\alpha<s$ and $0<\varepsilon<1$. It can be proved by using integration by parts and $A_{\mathcal{S}_{s}}(u) \ll u^{s}$. Thus, for $s \geq 4, X \leq x \leq 2 X$ and $X^{-1} \leq \varepsilon<1$,

$$
P_{\mathcal{B}_{s}}(x) \ll X^{(s-1) / 2}\left(\left|S_{+}(x)\right|+\left|S_{-}(x)\right|\right)+X^{s-1} \varepsilon,
$$

where

$$
S_{ \pm}(x)=\sum_{k \in Z_{*}^{s}} \alpha_{ \pm k}\|k\|^{-(s+1) / 2} \widehat{\delta}_{\varepsilon}(k) e^{2 \pi i x_{ \pm} H(k)} .
$$

This is the basic estimate of $P_{\mathcal{B}_{s}}(x)$. We remark that (12) remains true if $s=3$ (in this case (11) is used with $\alpha=s$; thus the bound $\varepsilon^{\alpha-s}$ in (11) has to be replaced by $|\log \varepsilon|)$. Moreover, the trivial estimate of $S_{ \pm}(x)$ together with (11) and the choice $\varepsilon=X^{-(s-1) /(s+1)}$ yields (1).

\section{The iterative estimation process}

Lemma 4. Let $\mathcal{B}=\mathcal{B}_{s}$ or $\mathcal{B}=\mathcal{B}_{s}^{*}$. Assume that for some $\gamma>2 s-3$ and $X \geq 1$,

$$
\int_{0}^{X}\left|P_{\mathcal{B}}(x)\right|^{2} d x \ll X^{\gamma} .
$$

Furthermore, let $R \geq U>0$ and $V \geq R^{(\gamma-1) / 2}$ be real parameters and set $M(U, V)=\#\left\{j \in \mathbb{Z}: 0 \leq j \leq R / U,\left|P_{\mathcal{B}}(R+j U)\right| \geq V\right\}$. 
Then

$$
M(U, V) \ll R^{\gamma} V^{-2}\left(R^{s-1} V^{-1}+U^{-1}\right) .
$$

Note that for $V \leq R^{(\gamma-1) / 2}$ the trivial bound $M(U, V) \ll R / U$ is sharper.

Proof. Since $A_{\mathcal{B}_{s}}(u)$ is increasing it follows that there is a constant $c>0$ such that for $R \leq v \leq u \leq 2 R$,

$$
P_{\mathcal{B}}(v)-P_{\mathcal{B}}(u) \leq \operatorname{vol}(\mathcal{B})\left(u^{s}-v^{s}\right) \leq \frac{c}{2}(u-v) R^{s-1} .
$$

Hence, if $t \in[R, 2 R]$ is a value with $\left|P_{\mathcal{B}}(t)\right| \geq V$, there exists an interval $I$ of length $V\left(c R^{s-1}\right)^{-1}$, containing $t$ as an endpoint, such that $\left|P_{\mathcal{B}}(u)\right| \geq V / 2$ for all $u \in I$. For all $j$ counted in $M(U, V)$ we choose (disjoint) intervals $J_{j}$ of length $\left|J_{j}\right|=\min \left(V\left(c R^{s-1}\right)^{-1}, U / 2\right)$ with $\left|P_{\mathcal{B}}(u)\right| \geq V / 2$ for all $u \in J_{j}$. Then trivially

$$
V^{2} M(U, V) \min \left(V R^{1-s}, U\right) \ll \int_{0}^{3 R}\left|P_{\mathcal{B}}(u)\right|^{2} d u \ll R^{\gamma}
$$

and

$$
M(U, V) \ll R^{\gamma} V^{-2} \max \left(V^{-1} R^{s-1}, U^{-1}\right) \ll R^{\gamma} V^{-2}\left(R^{s-1} V^{-1}+U^{-1}\right) .
$$

In the following we assume that (13) is true for $P_{\mathcal{B}_{s}^{*}}$. The basic estimate (12) is used to bound the mean square of $P_{\mathcal{B}_{s}}$. Since

$$
\int_{X}^{2 X} e^{2 \pi i \beta x} d x \ll \min \left(X,|\beta|^{-1}\right) \quad(\beta \in \mathbb{R}),
$$

it follows that

$$
\begin{aligned}
\int_{X}^{2 X} \mid & \left.S_{ \pm}(x)\right|^{2} d x \\
& \ll \sum_{k, m \in Z_{*}^{s}}(\|k\| \cdot\|m\|)^{-(s+1) / 2}\left|\widehat{\delta}_{\varepsilon}(k)\right| \cdot\left|\widehat{\delta}_{\varepsilon}(m)\right| \min \left(X,|H(k)-H(m)|^{-1}\right) .
\end{aligned}
$$

Hence by (12),

$$
\int_{X}^{2 X}\left|P_{\mathcal{B}_{s}}(x)\right|^{2} d x \ll X^{s-1}\left(X \Sigma_{1}+\Sigma_{2}\right)+X^{2 s-1} \varepsilon^{2},
$$

where

$$
\Sigma_{1}=\sum_{\substack{k, m \in Z_{*}^{s} \\|H(k)-H(m)|<X^{-1}}}(\|k\| \cdot\|m\|)^{-(s+1) / 2}\left|\widehat{\delta}_{\varepsilon}(k)\right| \cdot\left|\widehat{\delta}_{\varepsilon}(m)\right|
$$


and

$$
\Sigma_{2}=\sum_{\substack{k, m \in Z_{*}^{s} \\|H(k)-H(m)| \geq X^{-1}}}(\|k\| \cdot\|m\|)^{-(s+1) / 2}\left|\widehat{\delta}_{\varepsilon}(k)\right| \cdot\left|\widehat{\delta}_{\varepsilon}(m)\right| \cdot|H(k)-H(m)|^{-1} .
$$

To estimate $\Sigma_{1}$ and $\Sigma_{2}$ we build blocks of the form $R<H(k) \leq 2 R, R=2^{l}$, $l \geq 0$, and (in the second sum) $U \leq|H(k)-H(m)|<2 U, U=X^{-1} 2^{n}$, $n \geq 0$. In the first sum the summation condition implies $H(k) \asymp H(m) \asymp R$. Since $H(k) \asymp\|k\|$ it follows that with some constant $c>0$,

$$
\Sigma_{1} \ll \sum_{R=2^{l} \geq 1} R^{-s-1} \exp \left(-c(\varepsilon R)^{1 / 2}\right) N\left(R, X^{-1}\right)+1,
$$

where

$N(R, U)$

$$
=\#\left\{(k, m) \in \mathbb{Z}^{s \times s}: R<H(k) \leq 2 R, H(k)-U<H(m) \leq H(k)+U\right\} .
$$

In the second sum we find $H(k) \asymp H(m) \asymp R$ if $U \leq R / 4$, thus

$$
\begin{aligned}
\Sigma_{2} \ll & \sum_{R} \sum_{X^{-1} \leq U \leq R / 4} R^{-s-1} \exp \left(-c(\varepsilon R)^{1 / 2}\right) U^{-1} N(R, 2 U) \\
& +\sum_{R} R^{-(s+3) / 2} \exp \left(-c(\varepsilon R)^{1 / 2}\right) A_{\mathcal{B}_{s}^{*}}(2 R) \sum_{m \in Z_{*}^{s}}\|m\|^{-(s+1) / 2}\left|\widehat{\delta}_{\varepsilon}(m)\right| .
\end{aligned}
$$

By (11) the innermost sum of the second term is $O\left(\varepsilon^{-(s-1) / 2}\right)$. Since $A_{\mathcal{B}_{s}^{*}}(2 R) \ll R^{s}$ and

$$
\sum_{l \geq 0,\left(R=2^{l}\right)} R^{\alpha} \exp \left(-c(\varepsilon R)^{1 / 2}\right) \ll \begin{cases}\varepsilon^{-\alpha}, & \alpha>0, \\ |\log \varepsilon|, & \alpha=0,\end{cases}
$$

the whole second term is $O\left(\varepsilon^{2-s}\right)$. Hence

$$
\Sigma_{2} \ll \sum_{R} \sum_{X^{-1} \leq U \leq R} R^{-s-1} \exp \left(-c(\varepsilon R)^{1 / 2}\right) U^{-1} N(R, 2 U)+\varepsilon^{2-s}
$$

and by (14) and (15),

$$
\begin{aligned}
& \int_{X}^{2 X}\left|P_{\mathcal{B}_{s}}(x)\right|^{2} d x \\
& \ll X^{s-1} \sum_{R} \sum_{X^{-1} \leq U \leq R} R^{-s-1} \exp \left(-c(\varepsilon R)^{1 / 2}\right) U^{-1} N(R, 2 U) \\
& \quad+X^{s-1} \varepsilon^{2-s}+X^{2 s-1} \varepsilon^{2} .
\end{aligned}
$$

To estimate $N(R, U)$ we cover the interval $(R, 2 R]$ by intervals of the form 


$$
\begin{array}{r}
\left(R_{j}, R_{j+1}\right], R_{j}=R+j U, 0 \leq j<R / U+1, \text { and define }\left({ }^{3}\right) \\
K_{j}=\#\left\{k \in \mathbb{Z}^{s}: R_{j}<H(k) \leq R_{j+1}\right\} .
\end{array}
$$

Then by Cauchy's inequality,

$N(R, U)$

$$
\begin{aligned}
& \ll \sum_{j} \#\left\{(k, m) \in \mathbb{Z}^{s \times s}: R_{j}<H(k) \leq R_{j+1}, R_{j-1}<H(m) \leq R_{j+2}\right\} \\
& \ll \sum_{j} K_{j}\left(K_{j-1}+K_{j}+K_{j+1}\right) \ll \sum_{j<R / U+1} K_{j}^{2} .
\end{aligned}
$$

Since

$$
\begin{aligned}
K_{j}^{2} & =\left(A_{\mathcal{B}_{s}^{*}}(R+(j+1) U)-A_{\mathcal{B}_{s}^{*}}(R+j U)\right)^{2} \\
& \ll\left(R^{s-1} U+\left|P_{\mathcal{B}_{s}^{*}}(R+(j+1) U)\right|+\left|P_{\mathcal{B}_{s}^{*}}(R+j U)\right|\right)^{2} \\
& \ll R^{2 s-2} U^{2}+\left|P_{\mathcal{B}_{s}^{*}}(R+(j+1) U)\right|^{2}+\left|P_{\mathcal{B}_{s}^{*}}(R+j U)\right|^{2}
\end{aligned}
$$

we obtain

$$
N(R, U) \ll R^{2 s-1} U+\sum_{0 \leq j \ll R / U}\left|P_{\mathcal{B}_{s}^{*}}(R+j U)\right|^{2} .
$$

The remaining sum can be estimated by Lemma 4 . Set $V=R^{(\gamma-1) / 2} 2^{r} \ll$ $R^{s}, 0 \leq r \ll \log R$. Then

$$
\begin{aligned}
N(R, U) & \ll R^{2 s-1} U+R^{\gamma} U^{-1}+\sum_{V \geq R(\gamma-1) / 2} V^{2} M(U, V) \\
& \ll R^{2 s-1} U+R^{\gamma}(\log R) U^{-1}+R^{s+(\gamma-1) / 2} .
\end{aligned}
$$

Together with (16) and (17) this implies

$$
\begin{aligned}
\int_{X}^{2 X}\left|P_{\mathcal{B}_{s}}(x)\right| d x \ll & X^{s-1} \sum_{R} \sum_{X^{-1} \leq U \leq R} \exp \left(-c(\varepsilon R)^{1 / 2}\right) \\
& \times\left(R^{s-2}+R^{\gamma-s-1}(\log R) U^{-2}+R^{(\gamma-3) / 2} U^{-1}\right) \\
& +X^{s-1} \varepsilon^{2-s}+X^{2 s-1} \varepsilon^{2}
\end{aligned}
$$

$\left({ }^{3}\right)$ I learned this trick from M. N. Huxley during the conference on Analytic and Elementary Number Theory in Vienna, July 1996, which has been organized in honour of E. Hlawka's 80th birthday. Huxley used it to bound the mean square of the Hlawka zeta function of a planar convex body on a vertical line. 


$$
\begin{aligned}
\ll & X^{s-1} \sum_{R} \exp \left(-c(\varepsilon R)^{1 / 2}\right) \\
& \times\left(R^{s-2}(\log X+\log R)+R^{\gamma-s-1}(\log R) X^{2}+R^{(\gamma-3) / 2} X\right) \\
& +X^{s-1} \varepsilon^{2-s}+X^{2 s-1} \varepsilon^{2} \\
\ll & X^{s-1}(\log X) \varepsilon^{2-s}+X^{s+1}(\log X) \varepsilon^{s+1-\gamma}+X^{s} \varepsilon^{(3-\gamma) / 2}+X^{2 s-1} \varepsilon^{2} .
\end{aligned}
$$

Note that $\gamma-s-1>0$ and $\gamma-3>0$ if $s \geq 4$. In the case $s=3$ the term $X^{s+1} \log X$ is too large to give a nontrivial estimate. Balancing the second and the last term yields the optimal choice $\varepsilon=X^{-(s-2) /(\gamma-s+1)} \geq X^{-1}$. With this choice it is easy to check that the remaining two terms are of smaller order (use $\gamma>2 s-3$ ), hence

$$
\int_{X}^{2 X}\left|P_{\mathcal{B}_{s}}(x)\right|^{2} d x \ll X^{2 s-1-2(s-2) /(\gamma-s+1)} \log X .
$$

Summing over $X 2^{-j}, j \geq 1$, we have proved that for $s \geq 4$ the estimate

$$
\int_{0}^{X}\left|P_{\mathcal{B}_{s}^{*}}(x)\right|^{2} d x \ll X^{\gamma}
$$

with $\gamma>2 s-3$ implies the bound

$$
\int_{0}^{X}\left|P_{\mathcal{B}_{s}}(x)\right|^{2} d x \ll X^{2 s-1-2(s-2) /(\gamma-s+1)} \log X .
$$

Under the assumptions of our Theorem, Lemma 1 shows that we can interchange the rôles of $\mathcal{B}_{s}$ and $\mathcal{B}_{s}^{*}$. Hence, starting with the trivial bound $\gamma_{0}=2 s+1$ for the mean square of $P_{\mathcal{B}_{s}}(x)$ and $P_{\mathcal{B}_{s}^{*}}(x)$, the iteration

$$
\gamma_{n+1}=2 s-1-\frac{2(s-2)}{\gamma_{n}-s+1}
$$

produces better bounds. Since $\gamma_{n+1}<\gamma_{n}$ for $\gamma_{n}>2 s-3$ the iteration converges to its largest fixpoint. The two fixpoints are $2 s-3$ and $s+1$. Hence, for $s \geq 4, \lim _{n \rightarrow \infty} \gamma_{n}=2 s-3$. This proves the Theorem.

\section{References}

[1] R. N. Bhattacharya and R. R. Rao, Normal Approximation and Asymptotic Expansions, Wiley, New York, 1976.

[2] S. Bochner, Die Poissonsche Summenformel in mehreren Veränderlichen, Math. Ann. 106 (1932), 55-63.

[3] E. Hlawka, Über Integrale auf konvexen Körpern I, Monatsh. Math. 54 (1950), $1-36$.

[4] -, Über Integrale auf konvexen Körpern II, ibid., 81-99. 
[5] M. N. Huxley, Exponential sums and lattice points II, Proc. London Math. Soc. 66 (1993), 279-301.

[6] - , The mean lattice point discrepancy, Proc. Edinburgh Math. Soc. 38 (1995), $523-531$.

[7] A. E. Ingham, A note on Fourier transforms, J. London Math. Soc. 9 (1934), $29-32$.

[8] V. Jarník, Über Gitterpunkte in mehrdimensionalen Kugeln, Math. Ann. 100 (1928), 699-721.

[9] —, Über die Mittelwertsätze der Gitterpunktlehre, 5. Abhandlung, Časopis Pěst. Mat. 69 (1940), 148-174.

[10] I. Kátai, The number of lattice points in a circle, Ann. Univ. Sci. Budapest. Eötvös Sect. Math. 8 (1965), 39-60.

[11] E. Krätzel and W. G. Nowak, Lattice points in large convex bodies, Monatsh. Math. 112 (1991), 61-72.

[12] —, - Lattice points in large convex bodies II, Acta Arith. 62 (1992), 285-295.

[13] K. Leichtweiss, Konvexe Mengen, Springer, Berlin, 1980.

[14] W. G. Nowak, On the average order of the lattice rest of a convex planar domain, Proc. Cambridge Philos. Soc. 98 (1985), 1-4.

[15] - An $\Omega$-estimate for the lattice rest of a convex planar domain, Proc. Roy. Soc. Edinburgh Sect. A 100 (1985), 295-299.

[16] - On the lattice rest of a convex body in $\mathbb{R}^{s}$, Arch. Math. (Basel) 45 (1985), $284-288$.

[17] - On the lattice rest of a convex body in $\mathbb{R}^{s}, I I$, ibid. 47 (1986), 232-237.

[18] - On the lattice rest of a convex body in $\mathbb{R}^{s}$, III, Czechoslovak Math. J. 41 (116) (1991), 359-367.

[19] J. A. Thorpe, Elementary Topics in Differential Geometry, Springer, New York, 1979.

[20] A. Walfisz, Gitterpunkte in mehrdimensionalen Kugeln, Monografie Mat. 33, Polish Scientific Publ., Warszawa, 1957.

Institut für Statistik

Technische Universität Graz

A-8010 Graz, Austria

E-mail: mueller@stat.tu-graz.ac.at 